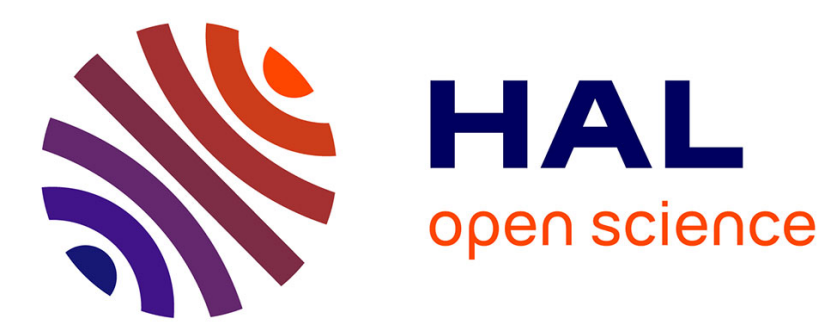

\title{
Acoustic characterization of Silica aerogel clamped plates for perfect absorption
}

A. Geslain, J.-P. Groby, V. Romero-Garcia, F. Cervera, J. Sánchez-Dehesa

\section{To cite this version:}

A. Geslain, J.-P. Groby, V. Romero-Garcia, F. Cervera, J. Sánchez-Dehesa. Acoustic characterization of Silica aerogel clamped plates for perfect absorption. Journal of Non-Crystalline Solids, 2018, 499, pp.283-288. 10.1016/j.jnoncrysol.2018.07.021 . hal-02366101

\section{HAL Id: hal-02366101 \\ https://hal.science/hal-02366101}

Submitted on 15 Nov 2019

HAL is a multi-disciplinary open access archive for the deposit and dissemination of scientific research documents, whether they are published or not. The documents may come from teaching and research institutions in France or abroad, or from public or private research centers.
L'archive ouverte pluridisciplinaire HAL, est destinée au dépôt et à la diffusion de documents scientifiques de niveau recherche, publiés ou non, émanant des établissements d'enseignement et de recherche français ou étrangers, des laboratoires publics ou privés. 


\title{
Acoustic characterization of Silica aerogel clamped plates for perfect absorption
}

\author{
A. Geslain \\ DRIVE EA1859, Univ. Bourgogne Franche Comté, F58000, Nevers France. \\ J.-P. Groby, V. Romero-García \\ Laboratoire d'Acoustique de l'Université du Mans, L'Université Nantes Angers Le Mans, Université du Mans, UMR CNRS 6613, Avenue Olivier \\ Messiaen, 72085 Le Mans, France. \\ F. Cervera, J. Sánchez-Dehesa \\ Wave Phenomena Group, Departamento de Ingeniería Electrónica, Universitat Politècnica de València, Camino de vera s/n (Edificio $7 F)$, \\ E-46022 València, Spain
}

\begin{abstract}
A multiobjective optimization procedure is employed to retrieve the viscoelatic parameters of silica aerogel clamped plates. This retrieval method preserves the aerogel sample integrity and, in contrast to the existing ones, relies on the minimization of two different cost functions. The first one, namely $J_{1}$, is related to the reflective properties of clamped plates backed by a rigid cavity, while the second one, namely $J_{2}$, concerns both the reflectance and transmittance spectra measured in transmission configuration. The recovered parameters are in agreement with previously reported values in the literature. In addition, they are also supported by designing structures for perfect absorption (100\% of absorption), which has been validated experimentally. Aerogel plates can be therefore used as innovative building units of artificial structures for the broadband absorption of sound.
\end{abstract}

Keywords: Aerogel, Acoustics, Mechanical characterization, Genetic algorithm

\section{Introduction}

Silica aerogels have been mainly developed for thermal and acoustic insulation purposes [1,2]. Therefore, their manufacturing process has been widely studied to improve their bulk properties and they present an extremely low density [3], an ultra low thermal conductivity [4], and a subsonic sound velocity [5, 6]. The extremely low static density is directly related to their high porosities. The frame of silica aerogel effectively consists of an assembly of connected small cross-sections beam-like elements resulting from fused nanoparticles. This particular assembly additionally provides silica aerogel a very low elastic stiffness when compared to rigid silica structure of identical porosity [6]. Silica aerogels are then nanoporous lightweight materials [2]. Beyond these soft bulk material properties, silica aerogel plates or membranes are excellent candidates to design original acoustic metamaterials, because they exhibit subwavelength resonances and present efficient absorption capabilities [7].
Recently, membrane-type metamaterials have shown an increasing interest to control acoustic waves. While a single membrane presents negative mass density [8] as well as may be used as a perfect subwavelength absorber $[9,10]$, the periodic arrangement of plates combined with other kind of resonators presents single and double negativity [11]. More recently, periodic arrangement of clamped plates has been used to control the harmonic generation [12] and the solitary wave generation in the nonlinear acoustic regime [13].

The characterization of the elastic properties of the silica aerogel plates is therefore primordial to design efficient aerogel-based acoustic metamaterials. Two types of test have been yet proposed to characterize their elastic properties: mechanical destructive tests and ultrasonic non-destructive tests. On the one hand, Woignier et al. [14, 15, 16] used 3-point bending and uniaxial compression while Haj-Ali et al. [17] used digital image correlation technique to determine the mecanical behavior of aerogel. On the other hand, ultrasonic tests 

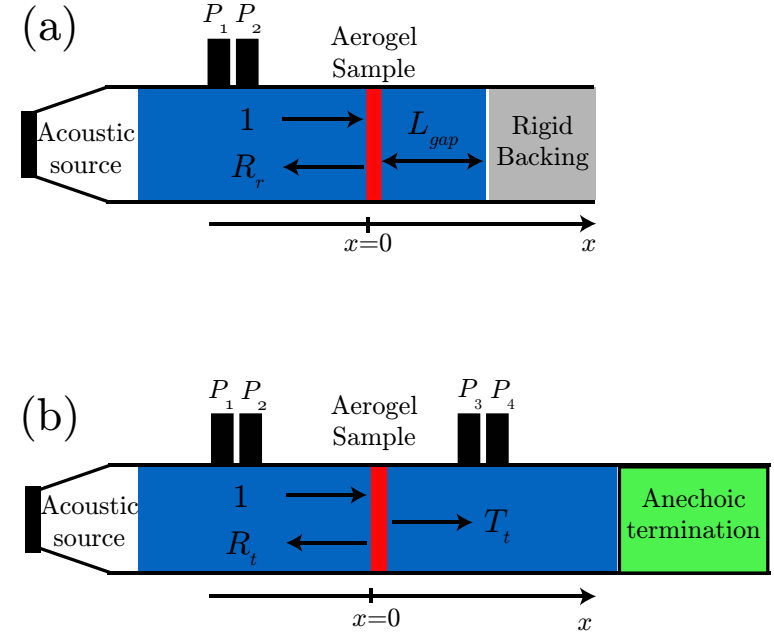

(c)

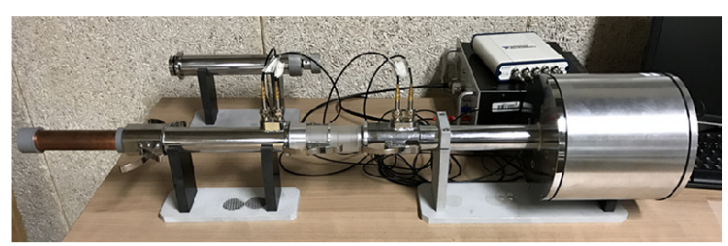

(d)

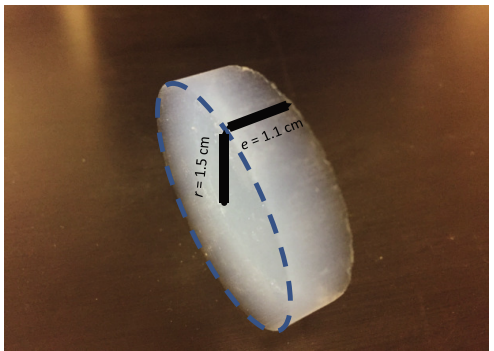

Figure 1: Reflection and transmission configurations and experimental set-up. (a) acoustic reflection configuration to determine reflection coefficient from the evaluated pressure $P_{1}, P_{2}$. (b) acoustic transmission problem to assess reflection and transmission coefficients from the pressures $P_{1}, P_{2}, P_{3}, P_{4}$. (c) Acoustic impedance tube mounted for the experimental characterization of the transmission problem. (d) Photograph of a Silica aerogel plate with thickness $e=1.1 \mathrm{~cm}$ and radius $r=1.5 \mathrm{~cm}$.

$[5,6,18,19]$ provide acoustic properties but are limited in terms of frequencies and by definition do not provide information in the audible regime.

This work aims at providing the viscoelastic properties of silica aerogel plates in the audible frequency range thanks to a novel signal processing method based on usual impedance tube measurements which have the advantage to preserve the sample integrity. The silica aerogel, being nanoporous, can be approximated by a viscoelastic material in this low frequency regime; i.e. in the audible frequency range. In this work we also show that clamped plates of silica aerogel can be used to design ultralight acoustic metamaterials for the perfect absorption of sound.

The article is divided in four Sections. In Section 2, the proposed method to retrieve the elastic properties is presented. A genetic optimization algorithm is used with two objective (or cost) functions to estimate Young modulus, loss factor, Poisson's ratio and mass density of the sample. The two objective functions arise from two complementary acoustic configurations: the reflection problem, where the aerogel membrane is backed with a rigid cavity, and the transmission problem. Section 3 make use of the recovered parameters to derive the perfect absorption (PA) condition [9, 20] (i.e. $\alpha=1$, where $\alpha$ is the acoustic absorption) when the silica-aerogel plate is backed by a rigid cavity of a specific length. By varying the cavity length from $0.5 \mathrm{~cm}$ to $6.5 \mathrm{~cm}$, the positions of both zeros and poles of the reflection coefficients are studied in the complex frequency domain.
Section 4 provides the concluding remarks and comments.

\section{Acoustic characterization of the silica aerogel plate mechanical properties}

The characterization of the viscoelastic properties of the silica aerogel plate is based on the analysis of data acquired in the two configurations shown in Fig. 1. In the first one, depicted in Fig. 1(a), the reflection coefficient of the aerogel plate when rigidly backed with an air cavity is measured. In the second one, depicted in Fig. 1(b), both the reflection and transmission coefficients of the aerogel plate in a transmission problem are measured. In addition, Fig. 1(c) shows a photograph of the experimental setup used for the characterization.

In this Section, we present the retrieval procedure which consists in minimizing the difference between the experimental and the theoretical coefficients.

\subsection{Direct modeling}

Assuming a Kirchhoff-Love plate [21], the transverse displacement $w_{s}$ satisfies the equation of motion

$$
D \nabla^{4} w_{s}+e \rho \frac{\partial^{2} w_{s}}{\partial t^{2}}=0
$$

where $e$ is the plate thickness, $\rho$ is the plate density and $D$ is the flexural rigidity. $D=\frac{E e^{3}}{12\left(1-v^{2}\right)}$, where $\mathrm{E}$ is the Young modulus and $v$ is the Poisson coefficient. Considering the temporal convention $e^{i \omega t}$, with 
$\omega$ the angular frequency, the silica aerogel viscoelastic behavior implies a complex Young modulus of the form $E=E_{r}(1+i \eta \omega)$, where $E_{r}$ is the real part of the Young modulus and $\eta$ is the loss factor. Assuming a sub-wavelength regime, the silica aerogel disk is considered as a punctual resonant element. The acoustic impedance $Z_{p}$ of a clamped circular cross-section plate was derived [7] and takes the form,

$$
Z_{p}=\frac{-i \omega \rho e}{S} \frac{I_{1}\left(k_{p} r\right) J_{0}\left(k_{p} r\right)+J_{1}\left(k_{p} r\right) I_{0}\left(k_{p} r\right)}{I_{1}\left(k_{p} r\right) J_{2}\left(k_{p} r\right)-J_{1}\left(k_{p} r\right) I_{2}\left(k_{p} r\right)},
$$

where $S=\pi r^{2}$ is the cross-section surface of the plate, $J_{n}$ and $I_{n}$ are the regular and modified Bessel functions of the first kind of order $n \in\{0,1,2\}$ and $k_{p}^{2}=\omega \sqrt{\rho e / D}$ is the plate wavenumber.

In the impedance tube, the aerogel clamped plate acoustically behaves as a point resonator mounted in series, implying sound velocity continuity and pressure ${ }^{142}$ discontinuity. Therefore, the clamped plate can be rep- ${ }^{143}$ resented by a matrix $M_{p}$ when using the transfer matrix ${ }_{144}$ method, [22] which reads as

$$
\left[M_{p}\right]=\left[\begin{array}{ll}
M_{p 11} & M_{p 12} \\
M_{p 21} & M_{p 22}
\end{array}\right]=\left[\begin{array}{cc}
1 & Z_{p} \\
0 & 1
\end{array}\right] .
$$

The reflection and transmission coefficients for this 149 symmetric and reciprocal transmission problem are then ${ }^{150}$ directly obtained from the elements of $M_{p}$ as, [22]

$$
\begin{aligned}
T & =\frac{2 e^{i k L}}{M_{p 11}+M_{p 12} / Z_{t}+Z_{t} M_{p 21}+M_{p 22}}, \\
R & =\frac{M_{p 11}+M_{p 12} / Z_{t}-Z_{t} M_{p 21}-M_{p 22}}{M_{p 11}+M_{p 12} / Z_{t}+Z_{t} M_{p 21}+M_{p 22}},
\end{aligned}
$$

where $Z_{t}=\rho_{t} c_{t} / S$ is the impedance of the surround- 158 ing medium, i.e. in our case the effective fluid occupy- 159 ing in the impedance tube, $S$ is the characteristic cross- 160 sectional area of the tube, $k$ is the wavenumber in the ${ }_{161}$ air and $L$ is the length of the sample, in our case $L=e .{ }^{162}$ Viscous and thermal losses are effectively accounted for 163 in the tube thanks to the formulae provided by Stinson 164 [23].

The aerogel plate system being symmetric and recip- 166 rocal, the modeled coefficients in the transmission problem are given by,

$$
\begin{aligned}
T_{\text {mod }}^{t} & =\frac{2}{2+Z_{p} / Z_{t}}, \\
R_{\text {mod }}^{t} & =\frac{Z_{p} / Z_{t}}{2+Z_{p} / Z_{t}} .
\end{aligned}
$$

In the reflection problem, the system is composed of 173 an aerogel plate in front of a closed cavity. In this case, 174 the transfer matrix method reads as,

$$
\left[\begin{array}{l}
P \\
V
\end{array}\right]_{x=0}=\left[M_{p}\right]\left[M_{c}\right]\left[\begin{array}{c}
P \\
0
\end{array}\right]_{x=L_{g a p}},
$$

with the propagation matrix $M_{c}$ given by

$$
\left[M_{c}\right]=\left[\begin{array}{cc}
\cos \left(k_{t} L_{\text {gap }}\right) & i Z_{t} \sin \left(k_{t} L_{\text {gap }}\right) \\
i \sin \left(k_{t} L_{\text {gap }}\right) / Z_{t} & \cos \left(k_{t} L_{\text {gap }}\right)
\end{array}\right],
$$

where $k_{t}$ is the wavenumber in the closed cavity (accounting for viscothermal losses) and $L_{\text {gap }}$ is the cavity length. The reflection coefficient is thus,

$$
R_{\text {mod }}^{r}=\frac{Z_{\text {syst }}-Z_{t}}{Z_{\text {syst }}+Z_{t}},
$$

where $Z_{s y s t}=P_{x=0} / V_{x=0}$ is the system impedance.

\subsection{Measurements of the reflection and transmission coefficients}

The reflection and transmission coefficients in each configuration are measured following the recovery of the scattering matrix elements as explained for example in Ref. [24]. In the reflection configuration, the two acoustic pressures, $P_{1}$ and $P_{2}$, are measured by the two microphones located upstream from the material to extract the frequency dependent experimental reflection coefficient $R_{e x p}^{r}(f)$ (where $f$ is the frequency). In the transmission problem, Fig. 1(b), the four acoustic pressures, $P_{1}, P_{2}, P_{3}$, and $P_{4}$, are measured by a pair of microphones located upstream and downstream from the material to obtain the experimental reflection and transmission coefficients $R_{\text {exp }}^{t}(f)$ and $T_{\text {exp }}^{t}(f)$ assuming the sample is symmetric.

The experimental setup is depicted in Fig. 1(c). The diameter of the impedance tube is $d_{t}=3 \mathrm{~cm}$. The experimental data are acquired with an excitation of 3000 sine functions equally spaced between $20 \mathrm{~Hz}$ and 6000 $\mathrm{Hz}$. Four 1/4 inch microphones are flush mounted, connected to a spectral analyser and measure the acoustic pressure. Each measurement is averaged 30 times to obtain the transfert function between the microphones. The aerogel samples are presented in Fig. 1(d).

\subsection{Retrieval procedure: Multiobjective optimization}

Decisions on optimal design in many scientific or engineering areas require a compromise between different objectives (or cost functions). It is therefore natural to look for the best solution to each of these cost functions. However, an improvement in one of the cost function can lead to a deterioration in the other ones if some cost functions are conflicting with the other. The absence of 

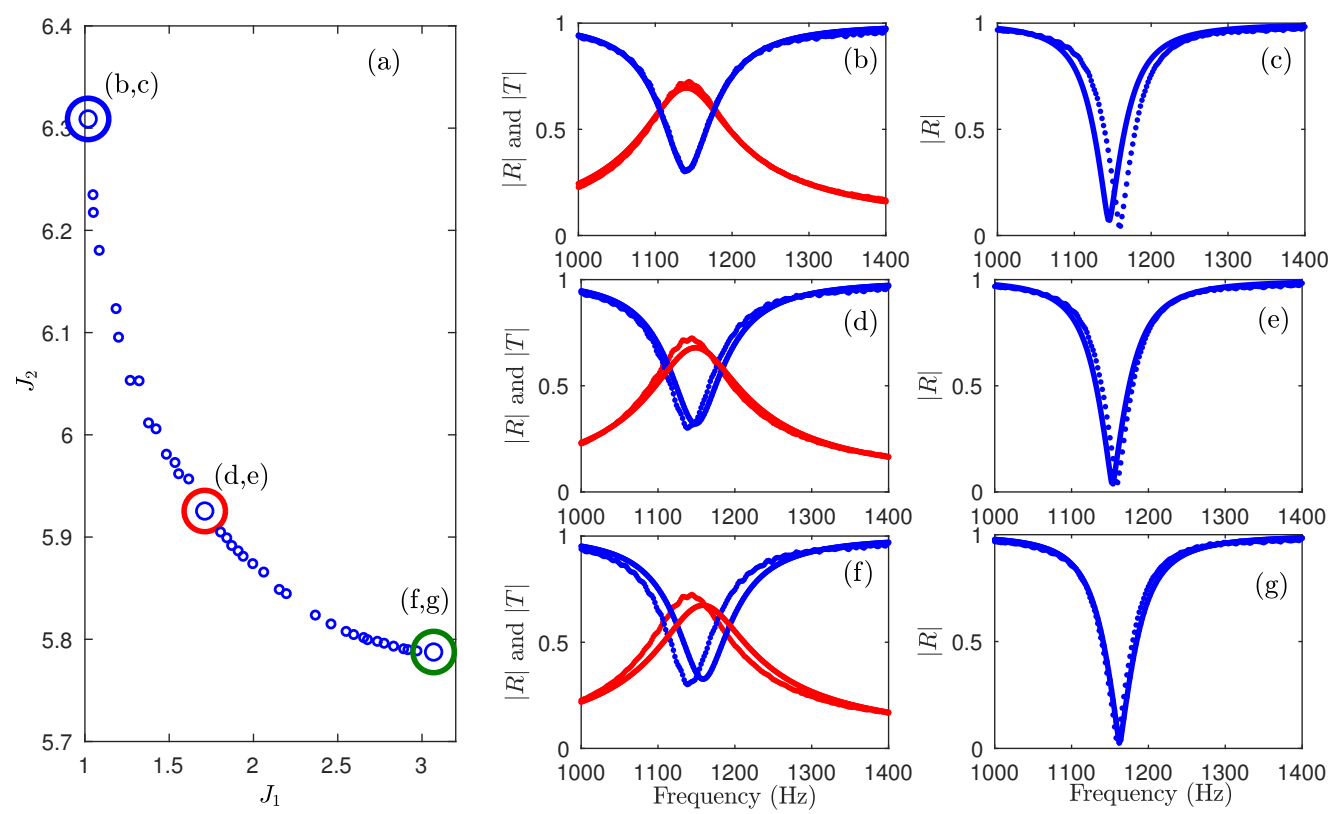

d)
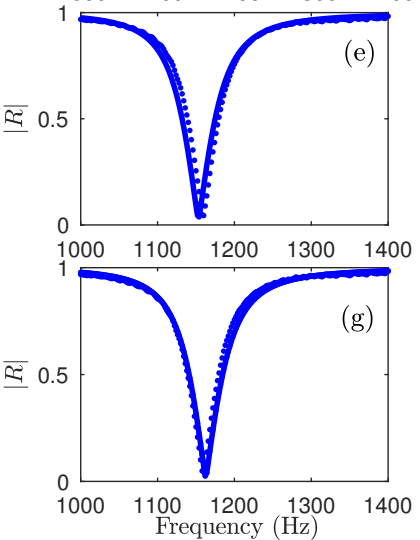

Figure 2: (a) Pareto front of the two objective functions $J_{1}$ and $J_{2}$. Big blue (red) [green] circle in (a) represents the Point 1 \{2\} [3] in Table 1. The reconstructed reflection and transmission coefficients for the transmission problem for this solution are shown in (b) $\{(\mathrm{d})\}[(\mathrm{f})]$ while the reconstructed reflection coefficient for the reflection problem is shown in (c) $\{(\mathrm{e})\}[(\mathrm{g})]$. Full lines represent the theoretical coefficients while dotted lines represents experimental results.

a single optimal solution is effectively a difficulty. Mul- 200 tiobjective optimization problems enable to avoid this ${ }_{201}$ issue. The optimal solution consists in a set $\Theta_{p}$ named 202 Pareto set [25]. The main characteristic of this set is ${ }_{203}$ that none of these elements are better than the others for ${ }_{204}$ some of the objectives. This means that all solutions are ${ }_{205}$ optimal in some sense.

In this Section we define the cost functions that are minimized by the multiobjective algorithm to retrieve the viscoelastic parameters of the aerogel plates, as well as the criteria chosen to identify the best solution and analyze the Pareto set. For the optimization problem, our approach is based on the use of evolutionary algorithms, which enable the simultaneous generation of several elements of the Pareto set in parallel and in a single run. Among these evolutionary algorithms, genetic algorithms, which are inspired by biological evolution (crossover, mutation, recombination and selection), are used [26]. The individuals of the genetic algorithm are 209 coded by chromosomes of the form, $\left(\rho, v, E_{r}, \eta\right)$. An 210 initial population (a set of possible solutions) evolves 211 by applying genetic operators that combine the charac- ${ }_{212}$ teristics of some of the individuals of the population. ${ }_{213}$ At each iteration, the population changes and adapts to ${ }_{214}$ converge to the optimal solution $\Theta_{p}$.

\subsubsection{The cost functions}

To retrieve the viscoelastic properties of the aerogel plate, two cost functions are defined. The two objective functions $J_{1}$ (transmission problem) and $J_{2}$ (reflection problem) are composed of the $L_{2}$ norms of the differences between the experimental and theoretical acoustic reflection and transmission coefficients over a range of frequencies centered on the resonance frequency system considered,

$$
\begin{aligned}
J_{1} & =\sum_{f=f_{1}}^{f_{2}}\left\|R_{\text {exp }}^{t}(f)-R_{\text {mod }}^{t}(\rho, v, E, \eta, f)\right\|^{2} \\
& +\sum_{f=f_{1}}^{f_{2}}\left\|T_{\text {exp }}^{t}(f)-T_{\text {mod }}^{t}(\rho, v, E, \eta, f)\right\|^{2}, \\
J_{2} & =\sum_{f=f_{1}}^{f_{2}}\left\|R_{\text {exp }}^{r}(f)-R_{\text {mod }}^{r}(\rho, v, E, \eta, f)\right\|^{2},
\end{aligned}
$$

where $R_{\text {exp }}^{t}, T_{\text {exp }}^{t}\left(R_{\text {mod }}^{t}, T_{\text {mod }}^{t}\right)$ are the experimental (respectively modeled) reflection and transmission coefficients in the transmission problem, and $R_{\text {exp }}^{r}\left(R_{\text {mod }}^{r}\right)$ is the experimental (respectively modeled) reflection coefficient in the reflection problem. In the summations $f_{1}=1124 \mathrm{~Hz}$ and $f_{2}=2324 \mathrm{~Hz}$. Each experimental coefficients were measured several times to check 
the measurement repeatability. The mechanical properties of the silica aerogel plate being independent of the length of the backing cavity, we chose without loss of generality and for convenience of our experimental setup, the gap thickness $L_{\text {gap }}=0.5 \mathrm{~cm}$ for the reflection problem [see Fig. 1(a)].

\subsubsection{Solutions: Pareto front}

Figure 2(a) shows the Pareto fronts of optimization procedure results. Each open circle in the Pareto front represents a solution with a different set of parameters $\left\{\rho, v, E_{r}, \eta\right\}$. The position of the solution in the Pareto front provides information on the accuracy of the retrieval procedure by using the two configurations previously described. The range of variation of the cost function $J_{2}$ is smaller than that of $J_{1}$ (see Fig. 2(a)). Therefore, the parameters are less sensitive to the reflection problem than to the transmission problem. In order to compare their accuracy, three solutions are given in Table 1 . These three solution are respectively highlighted in the Figure 2(a) with blue, red and green circles. Among all the solutions, the point $\left(J_{1}, J_{2}\right)=(1.027,6.308)\left[\left(J_{1}, J_{2}\right)=(3.077,5.786)\right]$ represents the best [wrong] solution for the objective $J_{1}, \quad 277$ i.e. for the transmission problem, and the worst [best] 278 solution for the objective $J_{2}$, i.e. for the reflection problem. We can clearly see in Figs. 2(b,c) [Figs. 2(f,g)] 279 that the differences between the reflection and transmis- 280 sion coefficients obtained for the transmission [reflec- 281 tion] problem are smaller than the ones obtained for the 282 reflection [transmission] problem for this solution. The 283 corresponding estimated parameters are summarized in 284 the Table 1 . The retrieval procedure provides relatively 285 identical values of $\left\{\rho, E_{r}, \eta\right\}$ at both points. This means 286 the transmission problem is enough to estimate these ${ }_{287}$ three parameters. In order to estimate $v$, the multiob- 288 jective optimization procedure accounting additionally 289 for the reflection problem should be considered. 290

In the opposite, the point $\left(J_{1}, J_{2}\right)=(1.719,5.924) 291$ represents the solution with the minimal distance to the 292 origin of coordinates, showing that the differences be- 293 tween the theory and the experiments for both problems 294 are similar (see Fig. 2(d,e)). As a consequence, the cri- 295 terium to choose the best solution is based on the mini- 296 mal distance to the origin of coordinates, and this point ${ }_{297}$ is chosen as the solution of our problem. The retrieved 298 viscoelastic parameters are given in Tab. 1 and their val- 299 ues are in agreement with others previously reported in 300 the literature [27, 28].

In order to further support the viscoelastic parame- 302 ters provided by the optimization problem, results of 303 reflection problems with different values of the cavity 304
Table 1: Optimal aerogel mechanical parameters of the three selected points in Fig. 2.

\begin{tabular}{c|c|c|c|c}
\hline \hline & $\begin{array}{c}\rho \\
\left(\mathrm{kg} . \mathrm{m}^{-3}\right)\end{array}$ & $v$ & $\begin{array}{c}E_{r} \\
(\mathrm{k} \mathrm{Pa})\end{array}$ & $\begin{array}{c}\eta \\
\left(10^{-6}\right)\end{array}$ \\
\hline Point 1 & 80 & 0.09 & 197.51 & 4.19 \\
\hline Point 2 & 80 & 0.12 & 197.92 & 4.47 \\
\hline Point 3 & 80 & 0.17 & 197.99 & 4.50 \\
\hline \hline
\end{tabular}

thickness $L_{\text {gap }}=[0.5,1,6.5] \mathrm{cm}$ are compared to measurements. Results of the absolute value and the phase of the reflection coefficients are respectively shown in Fig. 3(a) and 3(b). Continuous and dotted lines represent respectively the theoretical values and experimental results respectively. The theoretical predictions and experimental data are in good agreement, giving support to the viscoelastic parameters estimated by the proposed optimization procedure and also validating both the measurement and the model approach.

\section{Critical coupling condition and perfect absorp- tion}

The absorption properties of a system composed of the silica aerogel plate is studied making use of the previously estimated viscoelastic properties. The aerogel plate behaves as a viscoelastic clamped plate with intrinsic losses. The combination of an elastic plate with a back closed cavity, as shown in Fig. 1(a), can be analyzed as an open lossy resonator. In this case, the resonator presents two types of losses, the energy leakage of the open resonator and the intrinsic losses of the system. Recently, the design of perfect absorbers for acoustic wave has received an increasing interest $[10,9,29,20,30]$. The perfect absorption (PA) condition (i.e. $\alpha=1$, where $\alpha$ is the acoustic absorption coefficient) is related to the exact counterbalance between the energy leakage of the system and the dissipated energy by the inherent losses [20], which is known as the critical coupling condition. In this Section we show the efficiency of systems based on silica aerogel plates to perfectly absorb the acoustic energy.

In order to provide insights to the perfect absorption process, the reflection coefficient of an aerogel plate backed by a closed cavity is analyzed in the complex frequency plane for different cavity lengths. The cavity length $L_{\text {gap }}$ varies between $0.5 \mathrm{~cm}$ and $6.5 \mathrm{~cm}$. In the present problem, the eigenvalue of the scattering matrix reduces to the reflection coefficient. The measured 

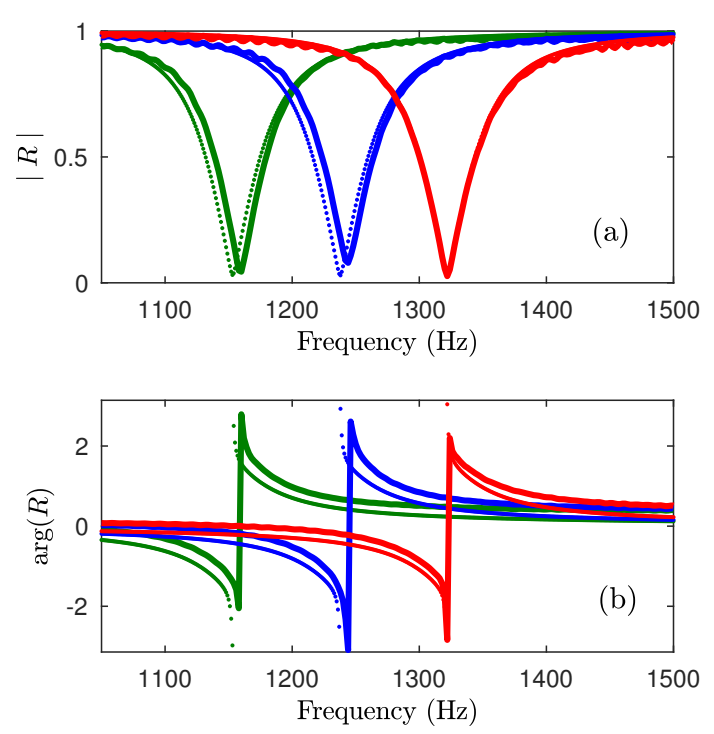

Figure 3: Comparison of the experimental reflection coefficient (circles) and the reconstructed reflection coefficient (solid lines) $\left[R_{\text {exp }}^{r}, R_{\text {mod }}^{r}\right]$ for the back cavity length $L_{\text {gap }}=[0.5,1,6.5] \mathrm{cm}[\mathrm{red}$, blue and green lines and symbols respectively]

- Aerogel mechanical parameters $\left[\rho=80 \mathrm{~kg} / \mathrm{m}^{3}\right.$, $\left.v=0.12, E_{r}=197.92 \mathrm{kPa}, \eta=4.4710^{-6}\right]$. (a) absolute value of the reflection coefficients. (b) phase of the reflection coefficient.

reflection coefficient corresponds to the one calculated along the real frequency axis. Therefore, the perfect absorption condition occurs in the complex frequency plane when the zero of the reflection coefficient crosses the real frequency axis $[9,20]$.

Figures 4(a), (b) and (c) show the reflection coefficient in the complex frequency plane for the configuration with $L_{\text {gap }}=[0.5,1,6.5] \mathrm{cm}$ respectively. For these three configurations, the zero of the reflection coefficient shifts at low frequencies when cavity length increases and, more importantly, it stands on the real frequency axis. Moreover, the zero of the reflection coefficient of each of these configurations is located exactly on the real frequency axis (red crosses in Fig. 4(a), meaning that all these configurations present perfect absorption. It is remarkable that the frequency at which perfect absorption is obtained is tunable in a broad range of frequencies by tuning $L_{\text {gap }}$.

Perfect absorption was experimentally verified. Red 350 and blue dashed lines in Fig. 4(d) show the theoretical ${ }_{337}$ reconstructed predictions and the experimental results 338 respectively. We can see that the frequency at which $3 з 9$ perfect absorption is obtained corresponds to the situ- 340 ation in which the zero of the reflection coefficients is 341

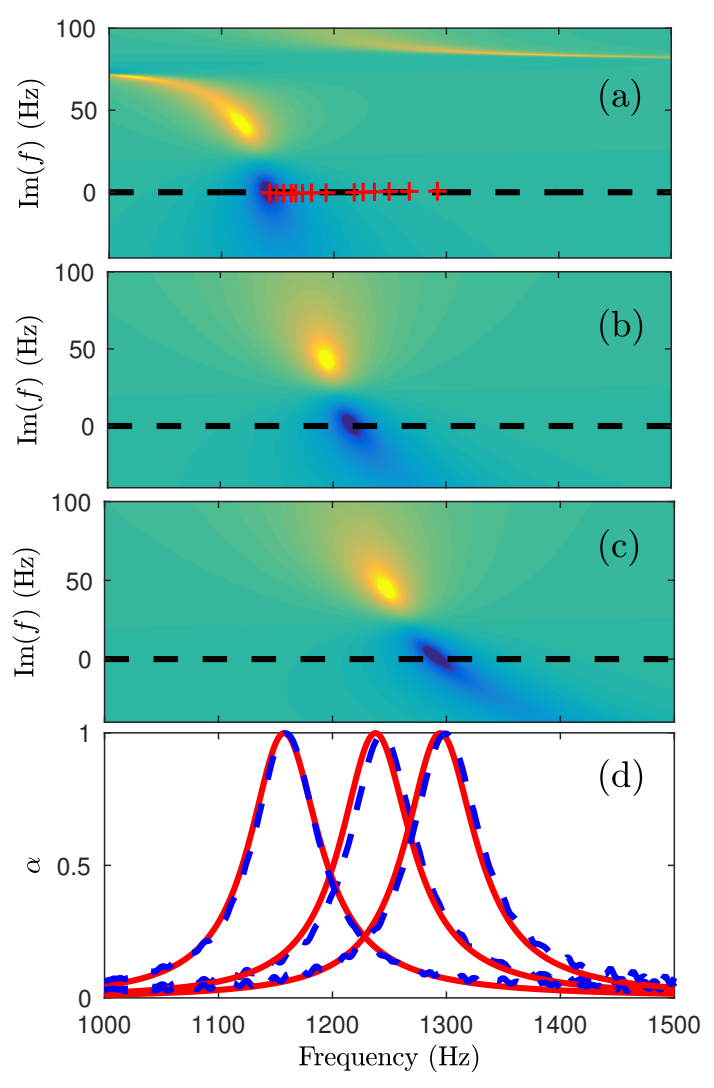

Figure 4: (a)-(c) Representation of the $\log _{10}\left(|R|^{2}\right.$ ) (color scale), in the complex frequency plane for the configurations with $L_{\text {gap }}=\{0.5,1,6.5\} \mathrm{cm}$ respectively. In (a) red crosses represent the complex frequency of the zero of $|R|$ in the complex frequency plane for the configurations $L_{\text {gap }}=$ $\{0.5,0.6,0.7,0.8,0.9,1.0,1.5,2.0,2.5,3.0,3.5,4.5,5.5,6.5\} \mathrm{cm}$.

(d) Comparison of the absorption coefficients, $\alpha$, for $L_{\text {gap }}=\{0.5,1,6.5\} \mathrm{cm}$ (in solid line theoretical reconstructed coefficients and in dashed line experimental results).

located on the real frequency axis. The agreement between the theory and the experiments is very good and makes aerogel plate backed by a closed cavity as an innovative artificial structures for acoustic absorption purpose.

\section{Summary}

A novel signal processing method for retrieving the viscoelastic properties of a silica aerogel clamped plate is presented. This method is based on a genetic algorithm optimization with two objective functions resulting from two acoustic configurations, a reflection problem and a transmission problem in an acoustic impedance tube. The two objective functions are com- 
posed of the $L_{2}$ norms of the differences between the 396 experimental and modeled acoustic problems around 397 the plate resonance frequency. The estimated aerogel ${ }^{398}$ viscoelastic properties are $\rho=80 \mathrm{~kg} / \mathrm{m}^{3}, v=0.12,{ }_{400}^{399}$ $E_{r}=197.92 \mathrm{kPa}, \eta=4.4710^{-6}$. These values are close 401 to those previously reported in the literature. The main ${ }^{402}$ advantages of the present method is that the aerogel sil- ${ }^{403}$ ica sample is undamaged and due to the genetic algo- ${ }_{405}$ rithm process the estimated viscoelastic properties sat- 406 isfy identically the transmission and reflection problems ${ }^{407}$ in the audible frequency range. Once the viscoelastic ${ }^{408}$ properties of the silica aerogel plate are characterized, 410 the perfect absorption condition (i.e. $\alpha=1$, with $\alpha$ is the 411 acoustic absorption) is derived for a system consisting ${ }^{412}$ in an aerogel plate backed by a rigid cavity. It is shown ${ }_{414}^{413}$ that such a system possess the ability to perfectly absorb $\quad 415$ sound over a wide range of cavity length, and therefore 416 over a wide range of possible frequency. The absorptive ${ }^{417}$ properties of aerogel are encouraging and can be applied ${ }_{419}^{418}$ to design more complex artificial structures (metasur- 420 faces) for the broadband absorption of sound.

\section{Acknowledgments}

This article is based upon work from COST Action DENORMS CA15125, supported by COST (European Cooperation in Science and Technology). The ${ }_{429}$ authors are grateful to the French ANR project Metaudi- ${ }^{430}$ ble (ANR-13-BS09-0003) and to RFI Le Mans Acous- ${ }^{431}$ tique (Région Pays de la Loire) PAVNat and Aerogel ${ }_{433}^{432}$ projects. F.C and J.S-D. acknowledge the partial sup- ${ }_{434}$ port by the Ministerio de Economía y Competitividad of 435 the Spanish Goverment and the European Union "Fondo ${ }^{436}$ Europeo de Desarrollo Regional (FEDER)" through ${ }_{438}^{437}$ project No. TEC2014-53088-C3-1-R.

[1] L. W. Hrubesh, "Aerogel applications", J. Non-Cryst. Solids 440 225, 335 (1998).

[2] M. Schmidt, F. Schwertfeger, "Applications for silica aerogel products", J. Non-Cryst. Solids 225, 364-368 (1998).

[3] M. Li, H. Jiang, D. Xu, O. Hai and W. Zheng, "Low density and hydrophobic silica aerogels dried under ambient pressure using a new co-precursor method", J. Non-Cryst. Solids 452, 187-193 (2016).

[4] Q. Xu, H. Ren, J. Zhu, Y. Bi, Y. Xu and L. Zhang, "Facile fabrication of graphite-doped silica aerogels with ultralow thermal conductivity by precise control", J. Non-Cryst. Solids 469, 14-18 (2017)

[5] V. Gibiat, O. Lefeuvre, T. Woignier, J. Pelous and J. Phalippou"Acoustic properties and potential applications of silica aerogels", J. Non-Cryst. Solids 186, 244-255 , (1995).

[6] M. Gronauer and J. Fricke, "Acoustic properties of microporous SiO2-aerogel”, Acta Acust united Ac. 59, 177 (1986).

[7] M. Guild, V.M. García-Chocano, J. Sánchez-Dehesa, T.P. Mar- 457 tin, D.C. Calvo, and G.J. Orris, "Aerogel as a Soft Acoustic Metamaterial for Airborne Sound", Phys. Rev. Appl. 5, 034012 (2016).
[8] Z. Yang, J. Mei, M. Yang, N. H. Chan and P. Sheng, "Membrane-Type Acoustic Metamaterial with Negative Dynamic Mass", Phys. Rev. Lett. 101, 204301 (2008).

[9] V. Romero-García, G. Theocharis, O. Richoux, A. Merkel, V. Tournat and V. Pagneux, "Perfect and broadband acoustic absorption by critically coupled sub-wavelength resonators", Sci. Rep. 6, 19519 (2016).

[10] G. Ma, M. Yang, S. Xiao, Z. Yang and P. Sheng, "Acoustic metasurface with hybrid resonances", Nat. Mat. 13, 873-878 (2014).

[11] S.H. Lee, C.M. Park, Y.M. Seo, Z.G. Wang and C.K. Kim, "Composite Acoustic Medium with Simultaneously Negative Density and Modulus”, Phys. Rev. Lett. 104, 054301 (2010).

[12] J.Zhang, V. Romero-García, G.Theocharis, O.Richoux, V. Achilleos and D. J. Frantzeskakis, "Second-Harmonic Generation in Membrane-Type Nonlinear Acoustic Metamaterials", Crystals 6 (2016).

[13] J.Zhang, V. Romero-García, G.Theocharis, O.Richoux, V. Achilleos and D. J. Frantzeskakis, "Bright and Gap Solitons in Membrane-Type Acoustic Metamaterials", Phys. Rev. E 96 (2017).

[14] A.H. Alaoui, T. Woignier, G.W. Scherer, J. Phalippou, "Comparison between flexural and uniaxial compression tests to measure the elastic modulus of silica aerogel", J. Non-Cryst. Solids 354, 4556-4561 (2008).

[15] T. Woignier, J. Pelous, J. Phalippou, R. Vacher and E. Courtens, "Elastic properties of silica aerogels", J. Non-Cryst. Solids 95\&96, 1197-1202 (1987)

[16] T. Woignier and J. Phalippou "Mechanical strength of silica aerogels", J. Non-Cryst. Solids 100, 404-408 (1988).

[17] R. Haj-Ali, R. Eliasi, V. Fourman, C. Tzur, G. Bar, E. Grossman,R. Verker, R. Gvishi,I. Gouzman and N. Eliaz, "Mechanical characterization of aerogel materials with digital image correlation", Micropor. Mesopor. Mat. 226, 44-52 (2016).

[18] T.E. Gomez Alvarez-Arenas, F.R. Montero de Espinosa, M. Moner-Girona, E. Rodriguez, A. Roig and E. Molins, "Viscoelasticity of silica aerogels at ultrasonic frequencies", Appl. Phys. Lett. 81, (2002).

[19] J. Groby, T. Schlief and J. Fricke"Ultrasonic evaluation of elastic properties of silica aerogels", Mater. Sci. Eng. 168, 235-238 , (1993).

[20] V. Romero-García, G. Theocharis and V. Pagneux, O. Richoux, "Use of complex frequency plane to design broadband and subwavelength absorbers", J. Acoust. Soc. Am. 139, 3395-3403 (2016).

[21] K.F. Graff, "Wave Motion in Elastic Solids ", Dover Publications New York, (1975).

[22] B.H. Song, J.S. Bolton, "A transfer-matrix approach for estimating the characteristic impedance and wave numbers of limp and rigid porous materials", J. Acoust. Soc. Am. 107, 1131 (2000).

[23] M. R. Stinson "The propagation of plane sound waves in narrow and wide circular tubes, and generalization to uniform tubes of arbitrary crosssectional shape", J. Acoust. Soc. Am. 89, 550-558 (1991).

[24] M. Niskanen, J.-P. Groby, A. Duclos, O. Dazel, J. C. Le Roux, N. Poulain, T. Huttunen and T. Lahivaara "Deterministic and statistical characterization of rigid frame porous materials from impedance tube measurements", J. Acoust. Soc. Am. 142, 24072418 (2017).

[25] Y. Censor, "Pareto Optimality in Multiobjective Problems", Appl. Math. Optimiz. 4, 41-59 (1977).

[26] T. Back., "Selective pressure in evolutionary algorithms: a characterization of selection mechanisms", Proceedings of the First IEEE Conference on Evolutionary Computation, IEEE Press, Orlando, 57 ?62 (1994).

[27] L Forest, V Gibiat, and T. Woignier, "Biot's theory of acoustic 
propagation in porous media applied to aerogels and alcogels", J. Non-Cryst. Solids, 225, 287 (1998).

[28] J. Gross, G. Reichenauer, J. Fricke, "Mechanical properties of $\mathrm{SiO} 2$ aerogels", J. Phys. D 21, 1447 (1988).

465 [29] N. Jiménez, W. Huang, V. Romero-García, V. Pagneux, J.P. Groby "Ultra-thin metamaterial for perfect and quasiomnidirectional sound absorption", Appl. Phys. Lett. 109, 121902 (2016).

[30] N. Jiménez, V. Romero-García, V. Pagneux, J.-P. Groby "Rainbow-trapping absorbers: Broadband, perfect and asymmetric sound absorption by subwavelength panels for transmission problems", Sci. Rep. 7, 13595 (2017). 